\title{
Conceptualización del diagnóstico en Trabajo Social: necesidades sociales básicas
}

\author{
Social work diagnosis conceptualization: Basics social needs \\ Eduardo DíAz HERRÁIz \\ Universidad de Castilla-La Mancha \\ eduardo.diaz@uclm.es \\ Patricia FERNÁNDEZ DE CASTRO \\ Universidad de Castilla-La Mancha \\ patricia.fernandez@uclm.es
}

Recibido: $28 / 06 / 2012$

Revisado: 23/10/2012

Aceptado: 02/02/2013

Disponible on line: 20/12/2013

\begin{abstract}
Resumen
Ya desde las primeras obras de la literatura en Trabajo Social, el intento por conceptualizar y definir el objeto del diagnóstico social ha estado presente. La pretensión que nos ocupa es la revisión de la literatura sobre el diagnóstico social para tratar de hacer una propuesta de sistematización y conceptualización, que nos permita tener una referencia conceptual para el diagnóstico social, y que posibilite la homogeneidad de los conceptos y categorías que utiliza la disciplina del Trabajo Social.

Se establecen cuatro niveles de conceptualización del contenido del diagnóstico social, como acercamiento a la interpretación de las cuatro categorías de necesidades sociales básicas de referencia teórico-analítica. Así, cualquier diagnóstico social podría tener como referencia de análisis e interpretación la supervivencia e integridad, la inclusiónintegración social, la autonomía personal y social y la identidad personal y social.
\end{abstract}

Palabras clave: Diagnóstico social, necesidades sociales básicas, inclusión, autonomía, identidad.

\begin{abstract}
Since first social work books, the attempt to define the social diagnosis object has been present. We try to review the social work literature about social diagnosis to propose a systematization and conceptualization that allow to have a social diagnosis conceptual reference, which makes possible homogeneity concepts and categories that are used in Social Work discipline.

Four levels of conceptualization are been described about the content of social diagnosis, as approach to four basic social needs categories used as theoretical-analytical reference for any social diagnosis. Therefore, survival and integrity, social inclusion-integration, personal and social autonomy and personal and social identity are taken as conceptual-analytical reference for social diagnosis.
\end{abstract}

Keywords: Social diagnosis, basics social needs, integrity, inclusion, autonomy, identity.

Referencia normalizada: Díaz Herráiz, E., y Fernández de Castro, P. (2013): «Conceptualización del diagnóstico en Trabajo Social: necesidades sociales básicas». Cuadernos de Trabajo Social, 26(2): 431-443.

Sumario: Introducción. 1. Definición de diagnóstico social. 2. Objeto del diagnóstico social: necesidades sociales básicas. 3. Conceptualización y categorías de las necesidades sociales básicas. 4. Conclusiones. 5. Referencias bibliográficas.

\section{Introducción}

La pretensión de esta revisión teórica del diagnóstico en Trabajo Social se centra en debatir y establecer su objeto y aportar una propuesta de sistematización que contribuya a generar unas categorías diagnósticas homogéneas con una consistente base conceptual. Para ello, se analiza en la literatura sobre el concepto de diagnós- 
tico social, considerando que todo diagnóstico es una evaluación; es decir, una descripción, análisis y valoración de las necesidades sociales, lo que significa llevar a cabo la interpretación de una situación existente en comparación con un modelo ideal de referencia.

Contribuir al debate sobre ese modelo de referencia para el diagnóstico social es uno de los principales objetivos del artículo, se analizan las teorías de necesidades sociales, pues, consideramos que, aun no siendo propias del Trabajo Social, disponen de un formidable potencial como construcción teórica en la que basar el objeto disciplinar. Así, se establecen los requisitos y características del concepto de necesidades sociales básicas y se especifican las categorías de las mismas que constituirían la referencia para el diagnóstico social.

Entendemos que las necesidades sociales básicas, cuyo origen y naturaleza son sociales, se refieren a factores objetivos indispensables para la supervivencia e integridad psicofísica de los seres humanos y la reproducción social. De este modo, se definen cuatro categorías homogéneas de aquellas necesidades íntimamente relacionadas con la esencia de la intervención social.

Las cuatro categorías de necesidades sociales, que constituyen ese modelo de referencia para el diagnóstico social, es decir, aquellas que deberían encontrarse en equilibrio, son: integridad, inclusión, autonomía e identidad; de las que se concreta su definición, conceptualización y contenido en cada caso.

En segundo lugar, nos proponemos establecer la secuencia metodológica del diagnóstico social, considerando que el acercamiento a una situación de necesidad social supone el razonamiento teórico secuenciado, a partir del establecimiento de relaciones entre los hechos y evidencias disponibles, orientado hacia su comprensión causal y su interpretación conceptual. Así, se define el diagnóstico social como la construcción de una explicación teóricoconceptual de la situación de necesidad social. Es decir, conocer en profundidad un fenómeno y conceptualizarlo, llevándolo a una síntesis explicativa que permita comprender su esencia, sus componentes fundamentales e interpretar las relaciones entre los factores.

Por ello, se establece el proceso para construir un diagnóstico social a partir de una se- cuencia procedimental en cuatro niveles de conceptualización y acercamiento a las necesidades sociales, que son: la síntesis descriptiva, el análisis causal, la interpretación y conceptualización de las necesidades sociales y la evaluación y pronóstico.

En definitiva, consideramos que esta revisión puede favorecer el debate sobre el diagnóstico en la disciplina del Trabajo Social, aportando una referencia de categorías y conceptos homogéneos, tanto a nivel teórico como metodológico, pues, se especifican tanto las categorías teórico-analíticas para el diagnóstico, como el proceso metodológico secuenciado en cuatro niveles de acercamiento para la construcción de todo diagnóstico social.

\section{Definición de diagnóstico social}

El diagnóstico social es una de las materias del Trabajo Social cuya literatura es más exigua. $\mathrm{Su}$ desarrollo teórico, conceptual y metodológico precisa una mayor investigación y teorización (De Robertis, 2003), pues es: «un procedimiento poco tratado en la disciplina del Trabajo Social» (Escartín, Palomar y Suárez, 1997, p. 118). En España, las comunidades autónomas han generado sus propias herramientas de gestión y documentación que utilizan diferentes conceptos diagnósticos, dando lugar a una terminología muy heterogénea. Aunque han existido trabajos (Martín, 2003; Karls y O'Keefe, 2008; Gobierno Vasco, 2009), que intentan homogeneizar sus instrumentos, el consenso sobre el objeto y la conceptualización del diagnóstico social no parece estar cerca de haberse alcanzado.

El diagnóstico social es un elemento fundamental en el proceso metodológico del Trabajo Social, pues las intervenciones y los resultados dependen de una adecuada interpretación y definición conceptual de las causas de las necesidades sociales. Es necesario establecer una apropiada conceptualización teórica y metodológica que permita consensuar procedimientos, herramientas y conceptos del diagnóstico social en Trabajo Social (Escartín, Palomar y Suárez, 1997). Pretendemos aportar una revisión de la literatura y contribuir a conceptualizar el objeto del diagnóstico social para homogeneizar los conceptos y su base teórica.

El diagnóstico constituye la segunda fase del método básico del Trabajo Social en la que, 
según Colomer (1979), se expresa el juicio sintético e interpretativo que hace el trabajador social de la situación estructural o personal, con miras a señalar las causas de los problemas o conflictos a fin de establecer posibles hipótesis de trabajo o de intervención profesional. Efectivamente, forma parte de un proceso y cobra sentido dentro de éste. Su realización no será posible mientras no dispongamos de la información suficiente y de calidad (en cantidad y cualidad) que permita comprender las necesidades sociales y sus causas. Igualmente, se encuentra vinculado a la planificación de la intervención, cuya base de formulación debe ser el propio diagnóstico realizado. La realización de un diagnóstico social conceptual es un requisito de eficacia para cualquier intervención social planificada (García, 2008) que quiera interpretar exitosamente las necesidades sociales, sus componentes y las relaciones entre los factores personales y sociales que configuran esa necesidad social.

Las distintas definiciones sobre el diagnóstico social en la literatura del Trabajo Social nos servirán para enmarcar el sentido y concepto que se ha dado al diagnóstico social. $\mathrm{La}$ primera definición la aporta Richmond (1995), como el «intento de efectuar con la mayor precisión posible una definición de la situación y personalidad de un ser humano con alguna carencia social» (p. 37). En nuestro país, las definiciones del diagnóstico social entienden que se trata de una síntesis, interpretación y evaluación profesional de una situación (Rosell, 1990), que consiste en formular los problemas del usuario o del grupo, y las formas en las que los factores personales y ambientales afectaran a su situación (Rubí, 1992).

El propósito del diagnóstico social radicaría en establecer una síntesis descriptiva y una interpretación y evaluación de la carencia social o de la situación personal y social (Colomer, 1979). Así, el contenido del diagnóstico social lo precisan convenientemente las definiciones.

$\mathrm{Su}$ objeto resulta definido de forma más vaga, puesto que la mayor parte de las definiciones hacen referencias poco precisas al respecto. Las menciones al objeto del diagnóstico son en términos de «situación», «problemas», «conflictos»o «carencia social». Conceptos, que no se refieren a un constructo teórico ni afrontan el objeto del diagnóstico social, dificultando la alusión de forma específica e inequívoca a la parte de la realidad que ocupa al mismo.

El contenido diagnóstico pretende sintetizar, definir, interpretar y conceptualizar; pero su definición en el Trabajo Social debe hacer referencia no solo a su estructura o contenido, sino también a qué le es propio, a aquello que sintetiza, define, interpreta y conceptualiza.

Entendemos el diagnóstico social como «el proceso que sintetiza, interpreta y conceptualiza la naturaleza y magnitud de las necesidades sociales en sus efectos, génesis y causas personales y sociales». Lo hemos definido, en primer lugar, como un proceso, supone un conjunto de operaciones a realizar dentro del proceso metodológico, no siendo un fin en sí mismo. Así, no tendría sentido realizar un diagnóstico si no se fuera a realizar una intervención o si no dispusiésemos de suficiente información.

En segundo lugar, hemos descrito el contenido del diagnóstico como una síntesis e interpretación que sistematiza y sintetiza los datos disponibles para poder interpretar; pero coincidimos con García (2008) en que se trata más bien de una valoración e interpretación que de una sistematización de la información, y esta síntesis persigue la adecuada compresión y valoración ${ }^{1}$. Por tanto, la síntesis del diagnóstico requiere ya una cierta valoración y conceptualización de la información recogida.

En tercer lugar, el diagnóstico social es interpretativo y conceptual. Su esencia no es la recopilación de datos, sino su análisis para establecer relaciones entre los factores. La interpretación y conceptualización refieren a un esfuerzo cognitivo por elaborar una explicación plausible, detallada y organizada de conceptos a partir del establecimiento de relaciones y asociaciones lógicas entre los hechos disponibles.

Por último, hacemos referencia a que el objeto del diagnóstico son las necesidades sociales, como constructo teórico que permite con-

${ }^{1}$ En este sentido es interesante revisar la diferenciación entre valoración y diagnóstico que aporta García (2008), y aunque la síntesis es parte del diagnóstico, su finalidad es interpretativa y no consiste, por lo tanto, en una mera recopilación de datos. 
ceptualizar la realidad propia del Trabajo Social. Así, corresponde al diagnóstico social establecer la naturaleza, magnitud, efectos, génesis y causas de las necesidades sociales. Este definirá las necesidades sociales presentes, tratando de reconocer las causas, personales y sociales, su origen y fundamento, además de los posibles efectos que las mismas han provocado y su previsible evolución.

\section{Objeto del diagnóstico social: necesida- des sociales básicas}

Entendemos que todo diagnóstico es una evaluación, es decir, una descripción, análisis y valoración del fenómeno (Fantova, 2005); supone interpretar una situación existente en comparación con un modelo de referencia. Precisamente este modelo de referencia ocupa parte del artículo, ya que, la literatura existente al respecto no es abundante.

En la definición del objeto del Trabajo Social existe cierto consenso en que el foco de intervención se sitúa en la interrelación entre el individuo y la sociedad y el equilibrio en esta relación. Las tres grandes concepciones sobre el objeto del Trabajo Social (situación psicosocial, estructura social y necesidades-recursos) se ubicarían bajo dicha rúbrica. Sin embargo, este axioma adolece de un alto grado de ambigüedad.

El concepto teórico de necesidades sociales permite tomar en consideración tanto los aspectos individuales como estructurales que pueden generar y configurar las situaciones de necesidad social; además, permite integrarlos en la conceptualización de las situaciones sociales concretas. Teóricamente nos distanciamos, por lo tanto, del binomio necesidades-recursos (De las Heras y Cortajarena, 1979; Casado, 1987) como concepción del objeto del Trabajo Social, dado que los recursos sociales competen al marco disciplinar de los Servicios Sociales.

Pese a que la teoría existente sobre las necesidades sociales no es propia del Trabajo Social, estimamos que dispone de un formidable potencial como construcción teórica en la que basar el objeto disciplinar, aun siendo conscientes de ciertas dificultades teóricas. Especialmente dos de ellas son las que trataremos de solventar en este artículo. Primero, cuál es la definición de necesidad, y segundo, qué ne- cesidades serían la referencia para el diagnóstico social.

\subsection{Concepto de necesidades sociales básicas}

Es ineludible partir de una noción objetiva y universal de las necesidades sociales, es decir, que su definición no dependa de la subjetividad, pudiéndose demostrar, y que se produzca en todos los seres humanos con independencia de su condicionamiento cultural. Las necesidades sociales básicas estarían relacionadas con la integridad de la persona (Bunge, 1999), para diferenciarlas de las necesidades secundarias, que podrían basarse en preferencias o deseos y que no tienen ese carácter universal. Así lo entendió Keynes (1981) en su diferenciación entre necesidades absolutas, presentes en toda situación e individuo, y las necesidades relativas cuya satisfacción es imposible, ya que a nivel de vida más alto más elevadas serán.

La concepción que nos concierne es la de necesidades sociales básicas. Es decir, aquellas que tiene el ser humano como ser social, tienen un carácter básico y refieren a la supervivencia e integridad. Son los factores objetivos indispensables para la supervivencia y la integridad psicofísica de los seres humanos (Ballester, 1999). Al profundizar en los requisitos que el constructo teórico de necesidades sociales básicas debe poseer, consideraremos que hace referencia a los factores objetivos indispensables para el mantenimiento de la vida y la reproducción social. El origen y naturaleza de las necesidades básicas es social, puesto que constituyen un requisito indispensable para la plena integración y participación social (Doyal y Gough, 1994). La cobertura de las necesidades sociales básicas no es arbitraria, ya que su no cobertura supone un perjuicio para el individuo. No son contingentes ni relativas, no caducan cuando se satisfacen y, aun así, se seguirán necesitando. Tampoco son finitas ni se agotan porque nunca llegan a satisfacerse completamente.

La objetividad de la existencia de necesidades sociales básicas se fundamenta en que, a pesar de existir cierto condicionamiento cultural, en esencia existen de manera homogénea y sustancial, independientemente de la cultura en la que nos encontremos. Podemos decir, por ejemplo, que la integración social es una necesidad universal, transcultural y objetiva en 
cualquier cultura o contexto social, que no depende, en su concepción de básica, de nuestros deseos y está presente en todos los seres humanos; que necesitamos la integración social y la perseguimos, aun cuando exista ya cierto nivel de la misma. Por ende, es de naturaleza y origen sociales y su satisfacción únicamente puede producirse en la interacción y relación sociales.

\subsection{Necesidades sociales básicas de referen- cia para el diagnóstico social}

Una vez establecido el concepto teórico que nos servirá como referencia para el diagnóstico social, es necesario delimitar cuáles serían las necesidades sociales básicas de referencia para el diagnóstico social. Tomaremos en consideración los conceptos y categorías de necesidades sociales básicas de las distintas teorías, seleccionando aquellas similares desde diferentes perspectivas, que puedan servirnos como referencia para elaborar una clasificación de las necesidades sociales básicas. La más conocida es la clasificación realizada por Maslow (1985), que establecía una escala funcional de las necesidades humanas, que representaba gráficamente en una pirámide, diferenciando cinco grupos de necesidades básicas jerarquizadas funcionalmente. En la base, las necesidades fisiológicas; las necesidades de seguridad o pervivencia en el mundo; las necesidades de posesividad y amor; las necesidades de estima personal y autoconcepto y, en la cúspide, las necesidades de autodesarrollo. Interesante para nuestro análisis resulta su diferenciación entre necesidades de supervivencia, más egoístas en su cobertura, y metanecesidades, situadas en la parte alta de la pirámide, pues, las últimas tendrían un carácter social y convivencial. Lo que apoya nuestra tesis de que las necesidades básicas de referencia para el diagnóstico en Trabajo Social tienen un origen y naturaleza social.

La diferenciación establecida por Keynes (1981) entre necesidades absolutas y relativas nos ha servido para distinguir entre necesidades objetivas y deseos. Cuestión también resuelta teóricamente por Doyal y Gough (1994), en su teoría generalista de las necesidades humanas, para quienes la distinción entre necesidades y deseos, tiene que ver con lo objetivo y lo subjetivo. La diferencia entre necesida- des y deseos es la intencionalidad y lo que se necesita objetivamente no depende del pensamiento, ya que la necesidad está ligada a la autorreproducción personal y social. En conclusión, la necesidad social básica y objetiva remite a la supervivencia digna en una sociedad (Ballester, 1999). En su teoría, Doyal y Goug parten de la condición de agente social del ser humano, para entender que las necesidades básicas son: «condiciones previas universalizables para la participación social y la persecución de los fines propios» (p. 64), destacando la salud física y la autonomía personal como condiciones para alcanzar un nivel adecuado de integración social.

Encontramos dos concepciones importantes que van a servirnos para establecer los criterios del concepto y las categorías de las necesidades sociales básicas. Primero, su carácter social y, segundo, el concepto y las categorías de autonomía personal e integración social como necesidades sociales básicas indispensables para la pervivencia y autoreproducción social.

Otra propuesta interesante sobre necesidades sociales es la realizada por Galtung (1985) dentro de las teorías del desarrollo (Galtung y Wirak, 1973), cuando postula que existen dos requisitos en la definición de las necesidades: se refieren a las condiciones indispensables para la existencia y a las condiciones necesarias para la existencia duradera de la sociedad. En definitiva, inciden en nuestra intención de relacionar la plena integración social como necesidad básica para la reproducción personal y social, en la naturaleza social de las necesidades sociales y en la condición objetiva de las mismas. La clasificación de Galtung (1985) inicia desde las necesidades más elementales hasta las menos elementales, distinguiendo cuatro categorías: seguridad, bienestar, libertad e identidad. Las necesidades de seguridad remiten a necesidades de supervivencia, las necesidades de bienestar a necesidades de alimentación, abrigo, asistencia sanitaria, educación, etc.; las necesidades de seguridad estarían ligadas a la libertad de elección y expresión, y las necesidades de identidad a necesidades de pertenencia.

En este mismo sentido, la teoría de las necesidades humanas fundamentales (Max Neef, 1993) considera que estarían presentes en todas las culturas y periodos históricos. $\mathrm{Pa}$ - 
ra Max Neef, la calidad de vida de las personas sería el principal indicador del desarrollo y estaría determinada por la posibilidad que las personas tienen de satisfacer sus necesidades humanas fundamentales. Desde esta perspectiva, las necesidades conformarían un sistema interrelacionado en el que las nueve necesidades humanas fundamentales interactuarían unas con otras y serían: libertad, identidad, creación, ocio, participación, entendimiento, afecto, protección y subsistencia.

Del conjunto de las teorías podemos concretar cuatro áreas de necesidades sociales básicas presentes en todas ellas y que estarían íntimamente relacionadas con la esencia de la intervención social, y por lo tanto, que podrían constituir las necesidades sociales básicas de referencia para el diagnóstico social: la integridad y supervivencia, la integración social, la autonomía personal y la identidad personal y social serían la referencia y el objeto de conceptualización de cualquier diagnóstico social.

\section{Conceptualización y categorías de las ne- cesidades sociales básicas}

Estas categorías de necesidades sociales básicas nos permiten agrupar los conceptos de necesidades sociales específicas sobre las que se interviene en Trabajo Social en cuatro grandes grupos, según su naturaleza, bien sea relativa a la integridad o supervivencia, la inclusión social o bien a la autonomía e identidad personal o social. Trataremos de definir el concepto y los contenidos fundamentales de cada una de las cuatro categorías de necesidades sociales básicas establecidas, aunque sin la pretensión de enumerar de manera exhaustiva todos los posibles conceptos, cuestión que podría ser objeto de posteriores análisis.

\subsection{Integridad y supervivencia}

Con esta categoría nos referimos a los factores básicos elementales para la reproducción de la vida y la supervivencia en el mundo. Es decir, a aquellos aspectos generalmente materiales cuya inexistencia, privación o falta de cobertura ponen en peligro la continuidad de la vida humana. Así, nos referimos a unas necesidades tan básicas como la alimentación y nutrición, el abrigo y alojamiento, la suficiencia económica para el mantenimiento básico, la salubridad, salud física y psíquica y el acceso a los servicios sanitarios, o a las situaciones de falta de libertad, violencia o maltrato que ponen en peligro la supervivencia.

\subsection{Inclusión/integración social}

Quizá sea esta categoría, junto con la autonomía personal, sobre la que existe un mayor consenso en torno a qué constituye una necesidad social básica objeto de intervención en Trabajo Social (Laparra y Aguilar, 1997). La inclusión o integración social se caracterizaría, según Castel (1992), por un trabajo estable y por una inscripción relacional sólida, familiar y de vecindad. Por oposición, la falta de inclusión social llevaría a la exclusión social entendida como «la imposibilidad de procurarse un lugar estable en las formas dominantes de organización del trabajo y en los modos conocidos de pertenencia comunitaria» (Castel, 1997, p. 18).

La noción de exclusión social tiene, además, un componente estructural, aglutina la síntesis de tres elementos: una dimensión y efectos económicos; una dimensión cultural y una serie de procesos sociales relacionados con el trabajo, como principal componente de inserción social (Tezanos, 1999). La exclusión social connotaría cierta idea de regresión con respecto a estas tres dimensiones que, de forma estructural, tenderían a generar mecanismos que exceptúan de la vida económica y social, como consecuencia de un conjunto de posiciones deficitarias con respecto a elementos laborales, económicos, culturales, personales y sociales que apartan de la participación e integración social (Fernández y Díaz, 2004).

Entre la inclusión y la exclusión se ha descrito un continuum en el que se establecen diferentes zonas (Castel, 1992). La zona de de integración se caracterizaría por un trabajo estable y por una inscripción relacional sólida. La zona intermedia de vulnerabilidad constituiría una zona inestable, en la que las actividades laborales se situarían entre la precariedad, trabajos intermitentes y paro, mientras que los aspectos relacionales se ubicarían en la fragilidad de los soportes sociales. Por último, la zona de marginalidad o exclusión social refiere a la ausencia de trabajo y aislamiento social.

La inclusión social como categoría del diagnóstico social debe tomar en consideración tres aspectos que van a determinar la posición entre la inclusión-exclusión social. Pri- 
mero, el acceso garantizado a los derechos económicos, políticos y sociales y las posibilidades de participación efectiva en la esfera política. Segundo, la conexión y solidez de las redes de reciprocidad social. Por último, el espacio de la producción económica, especialmente del mercado de trabajo, pues, el empleo es la vía principal de obtención de ingresos, así como, una de las principales vías de producción identidad (Subirats, 2004).

La categoría diagnóstica de inclusión/integración social supondría detectar las necesidades básicas en relación al contexto socioambiental (sistemas de servicios públicos: salud y seguridad, legal, justicia, cultura, educación, familiar), y al funcionamiento y participación social (relaciones y redes sociales, familiares, laborales, de ocio e interacción personal, conducta y estatus social) (Hepworth, Rooney, y Larsen, 1996).

\subsection{Autonomía personal y social}

La autonomía es la capacidad de acción basada en la comprensión de si mismo y de la cultura, además, de la capacidad cognitiva y emocional del individuo. Es decir, el conjunto de aptitudes cognitivas, anímicas y sociales, así como el acceso garantizado a los medios para adquirir esas aptitudes que permiten tomar decisiones dentro de su cultura (Doyal y Gough, 1994). En definitiva, la autonomía personal consiste en la capacidad para funcionar competentemente como persona adulta, regirse por criterios propios y mantenerse de forma independiente. La falta de esas capacidades y aptitudes llevaría a la situación opuesta a la autonomía, esto es, a la dependencia. Situación en la que la persona no puede mantenerse de forma autónoma o no es competente para la vida independiente y/o la adopción de decisiones propias.

Diferenciaremos tres subcategorías dentro de la autonomía: autonomía física, psíquica o intelectual, autonomía personal y autonomía social. La concepción de la autonomía física y psíquica o intelectual entiende la autonomía como aquella capacidad para mantener una vida independiente, sin ayuda o vigilancia de otras personas, es decir, la capacidad de una persona para valerse por sí misma y realizar las actividades diarias de la vida. La concepción de la autonomía personal haría referencia a tres dimensiones (Arto, 1993; Covey, 2000). Primero, la autonomía cognitiva referida a la capacidad de regirse por criterios propios, sopesando la presión ejercida por las opiniones externas, orientada por una conciencia realista de las capacidades y recursos personales. Segundo, autonomía emocional entendida como habilidad para manejar los sentimientos y emociones propios y ajenos, discriminarlos unos y otros y utilizar esta información para guiar pensamientos y acciones y, tercero, la autonomía conductual como capacidad para tomar decisiones independientes y hacerse responsables de sus actuaciones.

La autonomía social es el conjunto de capacidades, competencias y habilidades sociales y de comunicación que posibilitan la interacción social. En definitiva, las capacidades básicas de interacción social, lenguaje, comunicación $\mathrm{y}$ control emocional, afectivo y conductual adecuados a los contextos, nivel cultural y formativo, administración de bienes, competencias y habilidades sociales para las relaciones interpersonales.

La categoría diagnóstica de la autonomía personal y social valoraría, por lo tanto, la capacidad para mantener una vida autónoma e independiente, la capacidad para la toma de decisiones responsables, el manejo de las emociones propias, las capacidades para la interacción social y el desenvolvimiento en el medio.

\subsection{Identidad personal y social}

La identidad es resultante de procesos sociales dinámicos y permanentes, como fenómeno social que surge en la dialéctica entre individuo y sociedad (Berger y Luckman, 1968). Es un producto de la relación dialéctica entre la realidad psicológica y la estructura social, relacionándose con la idea de que las personas se hacen sobre quienes son y sobre lo que tiene sentido (Giddens, 2002). Consideraremos dos subcategorías en la identidad como categoría diagnóstica en Trabajo Social: identidad personal e identidad social. La identidad personal consiste en el conjunto de ideas que tenemos acerca de cómo somos y cómo nos ve el mundo; no obstante, la identidad personal es un proceso de naturaleza social que únicamente se construye en interacción. La percepción y toma de conciencia de sí mismo se cimenta sobre la percepción de los demás, a partir de las ex- 
periencias vitales en interacción en el recorrido biográfico personal. Así, la identidad personal es un proceso subjetivo y socialmente construido, con un marcado carácter emocional, cuyos resultantes fundamentales son el autoconocimiento, la confianza y seguridad en sí mismo y la autoestima personal, como procesos de reconocimiento y valorización de la propia individualidad (Erikson, 1968). En la construcción de la identidad personal hay dos factores cardinales: la socialización previa y las experiencias vitales adquiridas en interacción social de la biografía personal en función de las circunstancias histórico-sociales del momento.

En el desarrollo de la identidad personal construimos y adquirimos el sentido de pertenencia a grupos culturales con los cuales se comparten características comunes. Este es el concepto de identidad social. Nos identificamos y diferenciamos de los otros dentro de una estructura social dada, en un momento histórico concreto. Podemos definir la identidad social como la «conciencia que tenemos las personas de pertenecer a un grupo o categoría social, unido a la valoración de dicha pertenencia» (Tajfel, 1981, p. 292). Si la identidad personal nos permite la diferenciación y la singularidad, la identidad social nos posibilita la vinculación, la pertenencia, la identificación y la comparación. Así, el concepto de identidad social nos permite comprender los mecanismos de discriminación social y prejuicio a partir del proceso de categorización social - según Tajfel- pero también la estructura que alienta la solidaridad y el altruismo, ya que se atribuye a la identidad social cierta capacidad de «despersonalización» al posibilitar que el individuo no actué únicamente en función de sus preferencias aisladas, sino como sujeto social y a favor del interés grupal (Turner y Killian, 1987).

Con respecto a los compontes que configuran, reconstruyen y condicionan los procesos de generación de la identidad, tanto personal como social podemos clasificarlos en tres grupos:

- Identidad biológica: imagen corporal, edad, sexualidad y reproducción.

- Identidad sociocultural: socialización, creencias, valores, prejuicios y mitos, género, religión, clase social, étnica, convivencia, fa- milia, vecindad, amistad, laboral, patrones culturales.

- Identidad psico-afectiva: emociones, deseos, apego, afecto, convivencia, género, sexualidad, autoconocimiento, autoconcepto $\mathrm{y}$ autoestima personal.

\section{Contenido del diagnóstico social}

Hemos establecido el objeto y naturaleza del diagnóstico en Trabajo Social, definiendo el diagnóstico social como «el proceso que sintetiza, interpreta y conceptualiza la naturaleza y magnitud de las necesidades sociales en sus efectos, génesis y causas personales y sociales».

Las fases del diagnóstico persiguen la comprensión e interpretación de las necesidades sociales, a partir de varios procesos de conceptualización de las evidencias sociales existentes.

El acercamiento a una situación de necesidad social supone el razonamiento teórico secuenciado, a partir del establecimiento de relaciones entre los hechos y evidencias disponibles, orientado hacia su comprensión causal y su interpretación conceptual. El esquema clásico de las fases del diagnóstico social (Hamilton, 1974) distingue tres niveles: un primer nivel que sintetiza descriptivamente la situación que se plantea; un segundo nivel para establecer las relaciones de causa efecto que condicionan la situación; y un tercer nivel de evaluación de los elementos potencialmente condicionantes. También, Rosell (1990) concibe la secuencia diagnóstica en tres momentos, la síntesis, interpretación y evaluación. Igualmente, Colomer (1979) incide en que la interpretación de datos incluye la descripción del problema, el análisis y relación de los factores condicionantes, principales núcleos de intervención y el pronóstico de la situación. Tampoco difiere la visión de García (2008) que establece las fases del diagnóstico social - como descriptiva, valorativa y predictiva - advirtiéndonos, sin embargo, de que el diagnóstico social supone en esencia la estimación ya que: «valorar supone constatar una serie de aspectos o circunstancias de una persona o familia, y relacionarlos con los parámetros que se han determinado a efectos de una calificación» (p. 14).

Esta observación sobre la esencia valorativa del diagnóstico social nos cautiva especialmente, dado que lo concebimos como una eva- 
luación, es decir, como una interpretación de la situación de necesidad existente en comparación con un modelo ideal de referencia. Por ello, hemos definido las cuatro áreas de necesidades sociales básicas que consideramos constituyen ese modelo de referencia para el diagnóstico social, es decir, aquellas necesidades sociales básicas que deberían encontrarse en equilibrio: Integridad, inclusión, autonomía e identidad.

Diagnosticar es construir una explicación teórico conceptual de la situación de necesidad social. Es decir, conocer en profundidad un fenómeno y conceptualizarlo, llevándolo a una síntesis explicativa que permita comprender su esencia, sus componentes fundamentales e interpretar las relaciones entre los factores. El proceso para llevar a cabo ese conocimiento riguroso de la necesidad social requiere que hagamos un acercamiento en tres niveles de conceptualización. No obstante, consideramos que la interpretación y valoración como fase del diagnóstico social requiere una mayor atención de la que se le ha prestado en la literatura, por ello, tomaremos este proceso como una fase más del diagnóstico social, siendo conscientes que forma parte de la fase valorativa e interpretativa, según la visión clásica del diagnóstico social.

El primer nivel de conceptualización nos permitiría seleccionar, entre la información disponible, las evidencias precisas y explicativas; el segundo nivel trataría de encontrar las relaciones entre las distintas variables y los factores que influyen en la situación y permitirían explicarla; el tercero consistiría en interpretar y definir las necesidades sociales básicas existentes, en sus causas y componentes, y, por último, el cuarto nivel perseguiría establecer el pronóstico de las necesidades sociales básicas.

Somos conscientes de que el análisis causal y la interpretación o valoración unidos constituyen la esencia del diagnóstico social y, en la práctica, no pueden separarse en la elaboración del diagnóstico. Por lo tanto, estaríamos estableciendo tres fases en la secuencia diagnóstica: síntesis descriptiva, interpretación causal y pronóstico. Simplemente a efectos didácticos, distinguiremos cuatro momentos de conceptualización en el diagnóstico en Trabajo Social: síntesis interpretativa, análisis causal, interpretación y conceptualización y pronóstico evaluativo.

\subsection{Primer nivel de conceptualización: sín- tesis descriptiva}

Para llevar a cabo este primer nivel del diagnóstico es imprescindible contar con información suficiente y de calidad, es decir, que sea explicativa de la situación de necesidad social, teniendo como precedente necesario un fructífero estudio de investigación. Trataríamos de establecer una síntesis descriptiva ordenada evolutivamente, es decir, en secuencia diacrónica que posibilite el acercamiento a la génesis histórica de los hechos objetivos, ya sean individuales o estructurales, así como de los subjetivos, esto es, de la interpretación y vivencia que las personas tienen de su situación. En definitiva, la síntesis descriptiva perseguiría disponer de todas las evidencias relevantes de la situación de necesidad social, habiendo despejado aquella información que no resulta significativa para comprender las necesidades sociales $\mathrm{y}$, por consiguiente, esta síntesis no consistiría en una mera recopilación de datos. Especial importancia tiene, en este momento, la ordenación histórica, tanto de los hechos objetivos como de los subjetivos, para posibilitar un acercamiento más efectivo a la génesis histórica de la necesidad y sus causas. Dado que se trata de una primera secuencia de conceptualización, la descripción no hace lógicamente, referencia a una descripción densa de toda la información disponible, se trata simplemente de sintetizar los componentes y evidencias suficientes y explicativos que permitirán el éxito en las siguientes secuencias diagnósticas.

Podremos afrontar con mayor éxito el resto de la secuencia diagnóstica, cuando dispongamos de una síntesis descriptiva que nos aporte evidencias, suficientes en cantidad y explicativas en calidad, sobre los hechos objetivos y subjetivos, la evolución histórica, el momento en que aparece y las posibles casusas y efectos, ya que de otro modo, bien por falta de evidencias bien por la escasa capacidad explicativa de las mismas, no podríamos comprender la situación de necesidad o sus causas.

\subsection{Segundo nivel de conceptualización: análisis causal}

El segundo nivel consiste en el análisis causal, o lo que es lo mismo, en la relación entre los factores y variables presentes para atribuir causalidad relacionándolos entre sí. Persigue des- 
entrañar las relaciones entre los factores existentes y conocer aquellas variables que actúan como causas o efectos de las necesidades sociales existentes. Relacionar los elementos presentes, atribuyendo causalidad a los distintos factores, aspira a conocer su comportamiento e inferir si cuando se los relaciona se comportan como variables independientes o como variables dependientes. Entre los factores que suelen tomarse en consideración en el análisis causal se encuentran tanto las posibles causas y efectos como la demanda, su origen, personas que la realizan, así como, el factor o factores desencadenantes (Rosell, 1990).

\subsection{Tercer nivel de conceptualización: in- terpretación y conceptualización de las ne- cesidades sociales}

La definición de la situación de necesidad supone conceptualizar la necesidad social que nos encontramos como una categoría de las necesidades sociales básicas. Es decir, especificar de qué necesidad social básica se trata, sus principales componentes y causas. En definitiva, mostrar, a partir de las evidencias existentes, la naturaleza y esencia de las necesidades sociales presentes, la interpretación de sus causas y componentes sobre la supervivencia y subsistencia o integridad biopsicosocial, inclusión/integración social, autonomía personal/social e identidad personal y social. En definitiva, un esfuerzo cognitivo para elaborar una explicación plausible, detallada y organizada de conceptos a partir del establecimiento de relaciones lógico-teóricas entre las evidencias disponibles.

\subsection{Cuarto nivel de conceptualización: eva-} luación y pronóstico de necesidades sociales Podemos separar este proceso en dos secuencias diferentes, aunque son complementarias: por un lado, la evaluación, que trata de conocer las posibilidades de cambio y cómo van a afectar a ese cambio los elementos presentes; por otro lado, el pronóstico cuya pretensión es avanzar sobre la evolución futura, que se puede esperar en las necesidades sociales básicas presentes en función de los factores existentes. Por consiguiente la evaluación se centraría en detectar los posibles factores de riesgo o protectores, mientras el pronóstico pretende prever la evolución futura de la situación de necesidad social en función de sus distintos componentes.

\section{Conclusiones}

Entendemos el diagnóstico social como «el proceso que sintetiza, interpreta y conceptualiza la naturaleza y magnitud de las necesidades sociales en sus efectos, génesis y causas personales y sociales». Su sentido se ubica en el conjunto del proceso metodológico, puesto que no constituye un fin en sí mismo y cuya esencia es interpretativa, dado que la simple síntesis de información ya debe suponer cierta valoración dirigida a la comprensión de las necesidades sociales. Por lo tanto, su esencia, lejos de ser meramente descriptiva o analítica, es la interpretación y conceptualización de las necesidades sociales desde referentes teóricos. Así, se refiere a un esfuerzo cognitivo por elaborar una explicación plausible, detallada y organizada de conceptos, a partir del establecimiento de relaciones y asociaciones lógicas entre las evidencias disponibles.

La referencia teórica, como modelo interpretativo-analítico para el diagnóstico social, nos lo aporta el concepto de necesidades sociales básicas. Esto es, aquellos factores objetivos indispensables para la supervivencia, integridad y reproducción social de los seres humanos que tienen un marcado carácter social. Abreviando las características y requisitos del concepto de necesidades sociales básicas, como constructo teórico objeto del diagnóstico social, diremos que la cobertura de las necesidades no es arbitraria, dado que, en caso de no ser cubiertas el individuo sufriría un perjuicio. Tampoco son contingentes, puesto que no son finitas, ni relativas, y aun estando cubiertas, seguiremos necesitándolas. Con independencia de que pueda existir cierto condicionamiento cultural, las necesidades sociales básicas existen, en esencia, de manera universal en cualquier cultura en la que nos encontremos.

De la revisión y sinopsis del conjunto de teorías sobre necesidades sociales podemos sintetizar cuatro áreas de necesidades sociales básicas que constituyen la esencia del diagnóstico social y que obedecen a los requisitos fijados para el constructo. Así, la integridad, inclusión, autonomía e identidad constituyen necesidades sociales básicas, universales y objetivas, con independencia del contexto cultural en el que nos encontrásemos, que, además, no dependen de deseos individuales, sino que tienen un origen social y cuya satisfacción úni- 
camente puede producirse en interacción social. La integridad, como categoría diagnóstica, iría referida a elementos básicos fundamentales para la reproducción de la vida y la supervivencia. Quedarían bajo está rúbrica necesidades primarias como la nutrición, el alojamiento, la salud física o a situaciones de violencia, falta de libertad o maltrato que ponen en peligro la supervivencia.

La segunda categoría de necesidades sociales básicas la constituye la inclusión social, que aglutina una dimensión económica, una dimensión relacional y una dimensión laboral. En definitiva, el acceso a derechos económicos, políticos y sociales, las posibilidades de participación social efectiva en las redes sociales y el acceso al mercado de trabajo, como vía principal de acceso a la integración social.

La autonomía, como tercera categoría de necesidades sociales básicas, comprende tres subcategorías: autonomía física, autonomía personal y autonomía social, cuya significación inmanente remite a la capacidad para desenvolverse competentemente y de manera soberana en el entorno. En definitiva, esta categoría compendia la capacidad de acción para ser independiente, basada en la comprensión sobre uno mismo y de la cultura, además, de la capacidad para la competencia cognitiva, emocional y social necesarias para la interacción social.

La cuarta categoría de necesidades sociales básicas es la identidad, producto de la relación dialéctica entre realidad psicológica y estructura social. Se subdivide en otras dos categorías: identidad personal e identidad social. La identidad personal se substancia en un proceso subjetivo, emocional y socialmente construido, cuyos efectos principales son el autoconocimiento, la seguridad y la autoestima personal. Por su lado, la identidad social se entiende como la construcción de pertenencia a grupos o categorías sociales dentro de una estructura social. Así, si la identidad personal nos permite la singularidad y la diferenciación, la identidad social nos facilita la vinculación y la pertenencia.

Por último, hemos abordado el contenido y el proceso del diagnóstico social a partir de cuatro niveles de conceptualización: la síntesis descriptiva, el análisis causal, la interpretación y conceptualización y la evaluación y pronóstico.
El primer nivel de conceptualización del diagnóstico social no consiste, a nuestro entender, en la recopilación de toda la información disponible, ya que ésta debería estar disponible en el estudio de investigación, sino que alude a una síntesis producto de la reflexión e interpretación sobre la base teórica y analítica del modelo de necesidades sociales básicas. Es decir, la selección de aquellas evidencias relevantes y explicativas ordenadas de forma diacrónica que permita comprender e interpretar las situaciones de necesidad social. El éxito de esta síntesis descriptiva pasa por disponer de evidencias, precisas en cantidad y explicativas en calidad, sobre los hechos objetivos y subjetivos, la génesis histórica y las posibles causas y efectos de las necesidades sociales, pues, de otro modo, por escasas evidencias o insuficiente relevancia explicativa, el fracaso en la comprensión de las necesidades sociales estaría asegurado.

El segundo nivel de conceptualización es el análisis causal, es decir, la atribución de causalidad a las distintas evidencias para inferir si éstas actúan como variables dependientes o independientes en el conjunto de relaciones que construyen las necesidades sociales.

La interpretación y conceptualización sería el tercero nivel de conceptualización y consiste en ubicar las situaciones particulares en el modelo de necesidades sociales básicas a partir de sus principales componentes y causas. Es decir, definir la situación de necesidad social particular desde sus causas y componentes a la conceptualización en las categorías de necesidades sociales básicas del modelo propuesto.

Por último, la evaluación y el pronóstico, serían el cuarto nivel de conceptualización del proceso metodológico del diagnóstico social. $\mathrm{Y}$ aunque coexisten, poseen un matiz diferenciador. La evaluación persigue identificar los factores protectores o de riesgo existentes y el pronóstico predice la evolución que se puede esperar en función de los mismos.

En definitiva, un diagnóstico teóricamente fundamentado y metodológicamente estructurado, a partir del establecimiento de relaciones lógico-teóricas secuenciadas, nos situaría en la esencia del conocer científico: predecir el comportamiento de las necesidades sociales y situarnos en una posición de ventaja para su resolución. 


\section{Referencias bibliográficas}

Arto, A. (1993). Psicología Evolutiva. Una propuesta educativa. Madrid: CCS.

Ballester, L. (1999). Las necesidades sociales. Teorías y conceptos básicos. Madrid: Síntesis.

Berger, P. L. y Luckman, T. (1968). La construcción social de la realidad. Buenos Aires: Amorrortu.

Bunge, M. (1999). Las ciencias sociales en discusión. Buenos Aires: Sudamericana.

Casado, D. (1987). Introducción a los Servicios Sociales. Madrid: Acebo.

Castel, R. (1992). La inserción y los nuevos retos de las intervenciones sociales. En F. Álvarez Uría, Marginación e inserción (pp. 25-36). Madrid: Endymion.

Castel, R. (1997). La exclusión social. En Actas IV encuentro internacional sobre Servicios Sociales (pp. 185-200). Valencia: Fundación Bancaixa.

Colomer, M. (1979). Método de Trabajo Social. Revista de Trabajo Social, 7, 20-30.

Covey, S. R. (2000). Los siete hábitos de la gente altamente efectiva. Barcelona: Paidós.

De las Heras, P. y Cortajarena, E. (1979). Introducción al Bienestar. Madrid: Federación Española de Asistentes Sociales.

De Robertis, C. (2003). Fundamentos del Trabajo Social. Valencia: Nau Libres.

Doyal, L. y Gough, I. (1994). Teoría de las necesidades humanas. Madrid: Icaria.

Erikson, E. (1968). Identidad, juventud y crisis. Buenos Aires: Paidós.

Escartín, M. J., Palomar, M. y Suárez, E. (1997). Introducción al Trabajo Social. Trabajo Social con individuos y familias. Alicante: Aguaclara.

Fantova, F. (2005). Manual para la gestión de la intervención social. Políticas, organizaciones y sistemas para la acción. Madrid: CCS.

Fernández, T. y Díaz, E. (2004). Personas mayores con ingresos escasos. En J. F. Tezanos, Tendencias en desigualdad y exclusión social. Tercer foro sobre tendencias sociales (pp. 409-458). Madrid: Síntesis.

Galtung, J. (1985). Sobre la paz. Barcelona: Fontamara.

Galtung, J. y Wirak, A. (1973). Humans needs, humans Rights, and the theories. París: UNESCO.

García, G. (2008). Reflexiones y utilidades sobre el diagnóstico y la programación de la intervención social. Ecos de Mary Richmond. Revista de politica social y servicios sociales, 83, 9-38.

Giddens, A. (2002). Sociología. Madrid: Alianza.

Gobierno Vasco. (2009). Diagnóstico social e intervención. Herramienta informática para el trabajo social. Vitoria: Servicio central de publicaciones del Gobierno Vasco.

Hamilton, G. (1974). Teoría y práctica del trabajo social de casos. México: Prensa Médica Mexicana.

Hepworth, D. H., Rooney, R. H. y Larsen, J. A. (1996). Direct social work practice: Theory and skills. Belmont, California: Wadsworth Pub Co.

Karls, J. M. y O'Keefe, M. E. (2008). Person in environment system manual. Washington, D. C.: Nasw Press.

Keynes, J. M. (1981). Las posibilidades económicas de nuestros nietos. Papeles de economía española, 6, 353-361.

Laparra, M. y Aguilar, M. (1997). Intervención social y exclusión social. Simposio Políticas sociales y exclusión social. Publicado en Dossier, 43 (pp. 91-117). Madrid: Cáritas española.

Martín, M. (2003). Manual de indicadores para el diagnóstico social. Disponible: http://www.diagnosticosocial.com/descarga.php (consultado el 10 de junio de 2011).

Maslow, A. H. (1985). Motivación y Personalidad. Barcelona: Sagitario.

Max Neef, M. A. (1993). Desarrollo a escala humana. Conceptos, aplicaciones y algunas reflexiones. Barcelona: Icaria.

Richmond, M. E. (1995). El caso social individual. El Diagnóstico Social. Madrid: Talasa.

Rosell, T. (1990). La entrevista en el Trabajo Social. Barcelona: Euge.

Rubí, C. (1992). Introducción al trabajo social. Barcelona: Llar del Llibre.

Subirats, J. (2004). Pobreza y exclusión social. Un análisis de la realidad española y europea. Barcelona: Fundación la Caixa. 
Tajfel, H. (1981). Grupos humanos y categorias sociales. Barcelona: Herder.

Tezanos, J. F. (1999). Tendencias de dualización y exclusión social en las sociedades tecnológicas avanzadas. Un marco para el análisis. En J. F. Tezanos, Tendencias en desigualdad y exclusión social. (pp. 11-54). Madrid: Sistema.

Tezanos, J. F. (2005). Tendencias en exclusión social y politicas de solidaridad. Octavo foro sobre tendencias sociales. Madrid: Sistema.

Turner, R. H. y Killian, L. M. (1987). Collective behaviour. Englewood Cliffs, N. J.: Prentice-Hall. Wandrei, K. y James, K. (2008). Person in environment. The PIE classification system for social functioning problems. (K. Wandrei, \& J. Karls, Edits.) Washington, D. C.: Nasw Press. 